\title{
Explicit Kummer varieties of hyperelliptic Jacobian threefolds
}

\author{
J. Steffen Müller
}

\begin{abstract}
We explicitly construct the Kummer variety associated to the Jacobian of a hyperelliptic curve of genus 3 that is defined over a field of characteristic not equal to 2 and has a rational Weierstrass point defined over the same field. We also construct homogeneous quartic polynomials on the Kummer variety and show that they represent the duplication map using results of Stoll.

Supplementary materials are available with this article.
\end{abstract}

\section{Introduction}

Let $A$ be an abelian variety of dimension $g \geqslant 1$, defined over a field $k$. The quotient of $A$ by the map which takes a point on $A$ to its inverse is a singular projective variety which can also be defined over $k$ and which can be embedded into $\mathbb{P}^{2^{g}-1}$. It is called the Kummer variety $K$ associated to $A$. The complex case is discussed in $[\mathbf{1}, \S 4.8]$.

If $A$ is an elliptic curve, then $K$ is simply the projective line over $k$. If $A$ is the Jacobian of a genus 2 curve $C$, then this construction yields the classical singular Kummer surface, which can be embedded as a projective hypersurface into $\mathbb{P}^{3}$. In the case where $\operatorname{char}(k) \neq 2$ and $C$ is given by an equation of the form $y^{2}=f(x)$, an embedding and defining equation of the Kummer surface have been constructed by Flynn [6], see also the exposition in Chapter 3 of the book [2] by Cassels and Flynn.

A particularly useful feature of the Kummer surface $K$ is that parts of the group structure on $A$ remain meaningful on $K$. For instance, translation by a 2-torsion point and multiplication by an integer $n$ on $A$ commute with negation and hence descend to well-defined maps on $K$. Moreover, one can define a pseudoaddition on $K$ using certain biquadratic forms $B_{i j}$. Formulas for duplication, translation by a 2-torsion point and pseudoaddition have been found by Flynn [6] and can be downloaded from http://people.maths.ox.ac.uk/flynn/genus2/ kummer/. Analogues of these for the case $\operatorname{char}(k)=2$ have been found by Duquesne [4] and, independently, a unified treatment for arbitrary $k$ and arbitrary defining equations $y^{2}+h(x) y=f(x)$ of $C$ has been presented by the present author in [13].

In the present paper we discuss analogues of some of these objects for Jacobians of hyperelliptic curves of genus 3 with a $k$-rational Weierstrass point. In this case an embedding of the Kummer variety into $\mathbb{P}^{7}$ has been constructed by Stubbs $[\mathbf{2 2}]$ and we recall his construction in $\S 2$. When $\operatorname{char}(k) \notin\{2,3,5\}$, we find a complete set of defining equations for the image of $K$ under this embedding in $\S 3$, at least for generic $C$; it turns out that in this case $K$ can be defined as the intersection of a quadric and 34 quartics in $\mathbb{P}^{7}$. The genericity assumption is not hard to check for a given curve and we conjecture that it is always satisfied.

In $\S 4$ we discuss traces of the group structure on the Jacobian that can be exhibited on the Kummer variety. Duquesne has constructed a matrix $W_{T}$ representing translation by a 2-torsion point $T \in A$ on $K$. It turns out that under a suitable genericity assumption, biquadratic forms $B_{i j}$ as in genus 2 cannot exist in our situation, see Proposition 4.1.

Received 10 December 2012; revised 25 April 2014.

2010 Mathematics Subject Classification 14K05 (primary), 14J30, 11G10, 11G50 (secondary).

I would like to acknowledge support from DFG through DFG grants STO 299/5-1 and KU 2359/2-1. 
However, building on the work of Duquesne, we construct homogeneous quartic polynomials $\delta_{1}, \ldots, \delta_{8} \in k\left[x_{1}, \ldots, x_{8}\right]$ which we conjectured to represent duplication on the Kummer variety in an earlier version of this article (and in [14]). This conjecture can now be proved using recent results of Stoll, see Theorem 4.5.

Stoll [21] has recently announced the construction of a different embedding $\xi$ of $K$ which is valid for arbitrary hyperelliptic genus 3 curves over fields of characteristic other than 2 ; see also the talk slides http://www.mathe2.uni-bayreuth.de/stoll/talks/Luminy2012.pdf. His embedding uses $\operatorname{Pic}^{4}(C)$, which is canonically isomorphic to $\operatorname{Pic}^{0}(C)$. This is analogous to the description of the dual Kummer surface associated to a genus 2 curve $C$ in terms of $\mathrm{Pic}^{3}(C)$ due to Cassels and Flynn $[\mathbf{2}, \S 4]$ (in dimension 3, the Kummer variety is self-dual). Moreover, Stoll has found defining equations for his embedding of the Kummer variety and, using representation-theoretic arguments, polynomials representing duplication and forms that can be used for pseudoaddition on $K$.

The formulas described in this paper are mostly too long to be reproduced here. They can be obtained at $[\mathbf{1 1}]$.

\subsection{Applications}

In genus 2, the Kummer surface has several arithmetic applications. The first application is an addition algorithm on $A$ that uses pseudoaddition on $K$, see [7]. Suppose that $k$ is a number field. Letting $h$ denote the naive height on $\mathbb{P}_{k}^{3}$, we get an induced naive height on the Jacobian which can be used to search for points in $A(k)$ of bounded height. Stoll's program j-points which uses this approach is available from http://www.mathe2. uni-bayreuth.de/stoll/programs/index.html. Furthermore, this naive height can be used to define and compute a canonical height $\hat{h}$ on $A$, which has numerous applications. See [7] for the construction and a first algorithm for the computation of $\hat{h}$. Several refinements are presented by Stoll in [19] and [20] and by the author in his thesis [14, Chapter 3]; see also the forthcoming paper $[\mathbf{1 6}]$.

In the genus 3 situation, we do not have an explicit description of pseudoaddition and hence we do not get a similar addition algorithm on $A$ using the results of the present paper (such an algorithm is given by Stoll in [21]). We do get a height function on $K$ by restriction of the standard height function on $\mathbb{P}^{7}$ and an induced naive height $h(P)=h(\kappa(P))$ on the Jacobian, where $\kappa: A \rightarrow K \hookrightarrow \mathbb{P}^{7}$ is discussed in $\S 2$. Using the defining equations of $K$ presented in $\S 3$, we get an algorithm that lists all $k$-rational points on $K$ or on $A$ up to a given height bound. We can also define a canonical height function

$$
\hat{h}(P)=\lim _{n \rightarrow \infty} 4^{-n} h\left(\kappa\left(2^{n} P\right)\right) .
$$

Using Theorem 4.5 one can prove analogues of several results from [20] and obtain an algorithm for the computation of $\hat{h}$ as in [20]. Further details are given in [14, $\S 4.4]$.

An explicit theory of heights on Jacobians of hyperelliptic curves of genus 3 has recently been developed by Stoll in [21] using his embedding $\xi$. Algorithms for the computation of the canonical height on Jacobians of hyperelliptic curves of any genus have been introduced by Holmes [8] and the present author [15]. However, these are not easily related to a naive height suitable for point searching, as is required by standard algorithms for saturation of finite index subgroups of the Mordell-Weil group, such as in [20]. A solution to this problem has recently been proposed by Holmes [9].

\section{Embedding the Kummer variety}

In his $\mathrm{PhD}$ thesis, Stubbs [22] has found an explicit embedding of the Kummer variety associated to the Jacobian of a hyperelliptic curve of genus 3 with a rational Weierstrass 
point into $\mathbb{P}^{7}$. In this section we recall this embedding, also providing formulas for the image on $K$ of non-generic points on the Jacobian.

We first fix some notation that we will use throughout this paper. Let $k$ denote a field of characteristic $\operatorname{char}(k) \neq 2$. We consider a hyperelliptic genus 3 curve $C$ over $k$, given by an equation

$$
Y^{2}=F(X, Z),
$$

in the weighted projective plane over $k$ with respective weights 1,4 and 1 assigned to the variables $X, Y$ and $Z$, where

$$
F(X, Z)=f_{0} Z^{8}+f_{1} X Z^{7}+f_{2} X^{2} Z^{6}+f_{3} X^{3} Z^{5}+f_{4} X^{4} Z^{4}+f_{5} X^{5} Z^{3}+f_{6} X^{6} Z^{2}+f_{7} X^{7} Z
$$

is a binary octic form in $k[X, Z]$ without multiple factors such that $\operatorname{deg}_{X}(F(X, Z))=7$. Then there is a unique point $\infty \in C$ whose $Z$-coordinate is zero. Every hyperelliptic genus 3 curve over $k$ with a $k$-rational Weierstrass point has an equation of the form (1) over $k$. We let $A$ denote the Jacobian of $C$ and we let $K$ denote its Kummer variety.

Every point $P \in A$ has a representative of the form

$$
\left(P_{1}\right)+\left(P_{2}\right)+\left(P_{3}\right)-3(\infty),
$$

where $P_{1}, P_{2}, P_{3} \in C$, and this representation is unique unless two of the $P_{i}$ are swapped by the hyperelliptic involution. We call a point $P \in A$ generic if $P$ can be represented by an unordered triple of points $\left(x_{1}, y_{1}, 1\right),\left(x_{2}, y_{2}, 1\right),\left(x_{3}, y_{3}, 1\right) \in C$ such that all $x_{i}$ are pairwise distinct.

Let $\Theta$ denote the theta divisor on $A$ with respect to the point $\infty$. It is well known that $\Theta$ is ample (cf. [17]) and that $2 \Theta$ is base point free (cf. [18, $\S$ II.6]). Hence, a basis of $\mathcal{L}(2 \Theta)$ gives an embedding of $K$. Note that $\mathcal{L}(2 \Theta)$ is equivalent to a certain space of symmetric functions on $C^{3}$ with restrictions on the poles as in [5] or [22]. Using this approach, Stubbs [22, Chapter 3] has found the following basis $\kappa_{1}, \ldots, \kappa_{8}$ of the space $\mathcal{L}(2 \Theta)$ :

$$
\begin{aligned}
\kappa_{1}= & 1, \\
\kappa_{2}= & x_{1}+x_{2}+x_{3}, \\
\kappa_{3}= & x_{1} x_{2}+x_{1} x_{3}+x_{2} x_{3}, \\
\kappa_{4}= & x_{1} x_{2} x_{3}, \\
\kappa_{5}= & b_{0}^{2}-f_{7} \kappa_{2}^{3}+f_{7} \kappa_{3} \kappa_{2}-f_{6} \kappa_{2}^{2}+3 f_{7} \kappa_{4}+2 f_{6} \kappa_{3}, \\
\kappa_{6}= & \kappa_{2} b_{0}^{2}+2 b_{0} b_{1}-f_{7} \kappa_{2}^{4}+3 f_{7} \kappa_{3} \kappa_{2}^{2}-f_{6} \kappa_{2}^{3}-f_{7} \kappa_{3}^{2}-f_{7} \kappa_{4} \kappa_{2}+2 f_{6} \kappa_{3} \kappa_{2}-f_{5} \kappa_{2}^{2} \\
& +2 f_{5} \kappa_{3}, \\
\kappa_{7}= & b_{1}^{2}-\kappa_{3} b_{0}^{2}+f_{7} \kappa_{3} \kappa_{2}^{3}-2 f_{7} \kappa_{3}^{2} \kappa_{2}+f_{6} \kappa_{3} \kappa_{2}^{2}+f_{7} \kappa_{4} \kappa_{3}-f_{6} \kappa_{3}^{2}+f_{5} \kappa_{3} \kappa_{2}-3 f_{5} \kappa_{4}, \\
\kappa_{8}= & \kappa_{2} b_{1}^{2}+2 \kappa_{3} b_{0} b_{1}+\kappa_{4} b_{0}^{2}+f_{7} \kappa_{3}^{2} \kappa_{2}^{2}-f_{7} \kappa_{2}^{3} \kappa_{4}+f_{7} \kappa_{2} \kappa_{3} \kappa_{4}-f_{7} \kappa_{3}^{3}+f_{6} \kappa_{3}^{2} \kappa_{2} \\
& -f_{6} \kappa_{4} \kappa_{2}^{2}+f_{5} \kappa_{3}^{2}-f_{5} \kappa_{4} \kappa_{2},
\end{aligned}
$$

where

$$
\begin{aligned}
b_{0} & =\left(x_{1} y_{2}-x_{2} y_{1}-x_{3} y_{2}+x_{3} y_{1}-x_{1} y_{3}+x_{2} y_{3}\right) / d, \\
b_{1} & =\left(x_{3}^{2} y_{2}-x_{3}^{2} y_{1}+x_{2}^{2} y_{1}+y_{3} x_{1}^{2}-y_{2} x_{1}^{2}-y_{3} x_{2}^{2}\right) / d, \\
d & =\left(x_{1}-x_{2}\right)\left(x_{1}-x_{3}\right)\left(x_{2}-x_{3}\right) .
\end{aligned}
$$

The map $\kappa: A \rightarrow \mathbb{P}^{7}$

$$
\kappa(P)=\left(\kappa_{1}(P), \ldots, \kappa_{8}(P)\right)
$$


defines an embedding of the Kummer variety into $\mathbb{P}^{7}$. We also provide formulas for the values of $\kappa(P)$ when $P$ is not generic, since no such formulas have been given by Stubbs. Following Mumford, we can represent any $P \in A(k)$ as a pair of homogeneous forms

$$
(A(X, Z), B(X, Z))
$$

where $A, B \in k[X, Z]$ have homogeneous degree 4 and 2 , respectively. If $P$ is generic, then $b_{0}$ and $b_{1}$ as defined above are simply the constant and linear coefficients of $B(X, 1) \in k[X]$.

Suppose that $P$ is generic, satisfying $x_{1} x_{2} x_{3} \neq 0$, and write the $\kappa_{i}(P)$ in terms of $z_{j}=$ $1 / x_{j}$ and $w_{j}=y_{j} / x_{j}, j \in\{1,2,3\}$. We then multiply by the common denominator and set $w_{3}=0$. This leads to the following formulas for $P$ having a unique representative of the form $\left(\left(x_{1}, y_{1}, 1\right)\right)+\left(\left(x_{2}, y_{2}, 1\right)\right)-2(\infty)$ and satisfying $x_{1} \neq x_{2}$ :

$$
\begin{aligned}
\kappa_{1}(P)= & 0, \\
\kappa_{2}(P)= & 1, \\
\kappa_{3}(P)= & x_{1}+x_{2}, \\
\kappa_{4}(P)= & x_{1} x_{2}, \\
\kappa_{5}(P)= & f_{5}+2 f_{6} \kappa_{3}(P)+f_{7} \kappa_{3}(P)^{2}+2 \kappa_{4}(P) f_{7}, \\
\kappa_{6}(P)= & f_{4}+f_{5} \kappa_{3}(P)-f_{7} \kappa_{4}(P) \kappa_{3}(P), \\
\kappa_{7}(P)= & -f_{4} \kappa_{3}(P)-3 f_{5} \kappa_{4}(P)+f_{7} \kappa_{4}(P)^{2}, \\
\kappa_{8}(P)= & \left(f_{3} \kappa_{3}(P)^{3}+f_{1} \kappa_{3}(P)+f_{2} \kappa_{3}(P)^{2}+2 f_{0}-2 y_{1} y_{2}+f_{4} \kappa_{4}(P) \kappa_{3}(P)^{2}-3 f_{3} \kappa_{4}(P) \kappa_{3}(P)\right. \\
& -2 f_{2} \kappa_{4}(P)+f_{5} \kappa_{4}(P)^{2} \kappa_{3}(P)-2 f_{4} \kappa_{4}(P)^{2}+f_{7} \kappa_{4}(P)^{3} \kappa_{3}(P) \\
& \left.+2 f_{6} \kappa_{4}(P)^{3}\right) /\left(x_{1}-x_{2}\right)^{2} .
\end{aligned}
$$

For the case $x_{1}=x_{2}$ it suffices to use the same $\kappa_{1}, \ldots, \kappa_{7}$ as above and

$$
\begin{aligned}
\kappa_{8}(P)= & b_{1}^{2}+\left(\kappa_{4}(P)-\kappa_{3}(P)^{2}\right)\left(-2 f_{7} \kappa_{4}(P) \kappa_{3}(P)\right. \\
& \left.-f_{6} \kappa_{4}(P)+f_{7} \kappa_{3}(P)^{3}+f_{6} \kappa_{3}(P)^{2}+f_{5} \kappa_{3}(P)+f_{4}\right),
\end{aligned}
$$

where $b_{1}$ is the linear coefficient of $B(X, 1) \in k[X]$ if the Mumford representation of $P$ is $(A, B)$.

Now consider points represented by

$$
\left(\left(x_{1}, y_{1}\right)\right)-(\infty)
$$

We first look at quotients of the form $\kappa_{i}(P) / \kappa_{5}(P)$, where $P$ is again assumed generic, and then take the limit $\left(x_{2}, y_{2}, 1\right) \rightarrow\left(x_{3},-y_{3}, 1\right)$. The result is

$$
\kappa(P)=\left(0,0,0,0,1,-x_{1}, x_{1}^{2}, x_{1}^{3}\right) .
$$

A similar argument shows that we have

$$
\kappa(O)=(0,0,0,0,0,0,0,1)
$$

where $O \in A$ is the identity element.

If $P \in A$, then we say that $x=\left(x_{1}, \ldots, x_{8}\right) \in \mathbb{A}^{8}$ is a set of Kummer coordinates for $P$ if $\kappa(P)=\left(x_{1}: \ldots: x_{8}\right)$. We set

$$
K_{\mathbb{A}}:=\left\{\left(x_{1}, \ldots, x_{8}\right) \in \mathbb{A}^{8}: \exists Q \in K \text { such that } Q=\left(x_{1}: \ldots: x_{8}\right)\right\} .
$$

Remark 2.1. In the general case $\operatorname{deg}_{X} F=8$ Stubbs constructs functions analogous to the functions $\kappa_{i}$. However, these do not give an embedding of the Kummer variety, since not all points on $A$ can be represented by unordered triples of points on $C$. See $[\mathbf{2 2}, \S 3.8]$ for a discussion. 
REMARK 2.2. As discussed in the introduction, Stoll [21] has recently constructed an embedding $\xi=\left(\xi_{1}, \xi_{2}, \xi_{3}, \xi_{4}, \xi_{5}, \xi_{6}, \xi_{7}, \xi_{8}\right)$ of the Kummer variety into $\mathbb{P}^{7}$ which is valid for arbitrary hyperelliptic curves $C: Y^{2}=F(X, Z)$ of genus 3 defined over a field of characteristic other than 2. When $\operatorname{deg}_{X} F=7$, his embedding is related to Stubbs' embedding $\kappa$ as follows:

$$
\begin{aligned}
& \xi_{1}=\kappa_{1} \\
& \xi_{2}=-f_{7} \kappa_{2} \\
& \xi_{3}=\quad f_{7} \kappa_{3} \\
& \xi_{4}=\quad-f_{7} \kappa_{4} \\
& \xi_{5}=f_{4} \kappa_{1}+f_{5} \kappa_{2}+2 f_{6} \kappa_{3}+3 f_{7} \kappa_{4}-\kappa_{5} \\
& \xi_{6}=f_{3} \kappa_{1}+f_{4} \kappa_{2}+f_{5} \kappa_{3}-\kappa_{6} \\
& \xi_{7}=f_{2} \kappa_{1} \quad-f_{4} \kappa_{3}-3 f_{5} \kappa_{4} \quad-\kappa_{7} \\
& \xi_{8}=\quad-f_{2} f_{7} \kappa_{2}-f_{4} f_{7} \kappa_{3}-f_{4} f_{7} \kappa_{4}+f_{7} \kappa_{8}
\end{aligned}
$$

\section{Defining equations for the Kummer variety}

In this section we compute defining equations for the Kummer variety $K$, embedded into $\mathbb{P}^{7}$ as in the previous section.

The following result seems to be well known to experts in algebraic geometry, but no proof seems to exist in the literature. The proof given here was suggested by Tzanko Matev.

Proposition 3.1. Let $A$ be a Jacobian variety of dimension $g \geqslant 2$ defined over an arbitrary field and let $\Theta$ be a theta divisor on $A$. If $\kappa_{1}, \ldots, \kappa_{2^{g}}$ is a basis for $\mathcal{L}(2 \Theta)$ and if $\kappa=\left(\kappa_{1}, \ldots, \kappa_{2^{g}}\right): A \rightarrow \mathbb{P}^{2^{g}-1}$, then the image $\kappa(A)$ can be described as an intersection of quartics.

Proof. Let $\mathcal{Q}=\left\{q_{1}, \ldots, q_{m}\right\}$ denote the set of monic quadratic monomials in $\kappa_{i}$, where $m=\left(\begin{array}{c}2^{g}+1 \\ 2\end{array}\right)$ and we assume, without loss of generality, that $\left\{q_{1}, \ldots, q_{d}\right\}$ is linearly independent in the space $\mathbb{Q}\left(f_{0}, \ldots, f_{7}\right)\left[q_{1}, \ldots, q_{m}\right]$, where $d \leqslant m$ is the dimension of the space generated by the elements of $\mathcal{Q}$.

Let $\iota$ denote the 2 -uple embedding of $\mathbb{P}^{2^{g}-1}$ into $\mathbb{P}^{m-1}$ such that for $P \in A$ we have

$$
\iota_{i}(\kappa(P))=q_{i}(P) \quad \text { for all } i \in\{1, \ldots, m\} .
$$

Then there are $m-d$ linear relations on the image of $K=\kappa(A)$ under $\iota$. Now consider an embedding $\beta: A \hookrightarrow \mathbb{P}^{4^{g}-1}$ given by a basis of $\mathcal{L}(4 \Theta)$ whose first $d$ elements are equal to $q_{1}, \ldots, q_{d}$. Then we have a commutative diagram

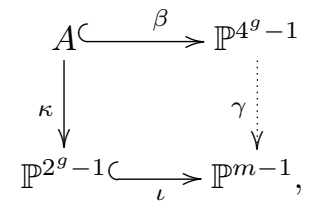

where $\gamma$ is a rational map defined as follows: if $z=\left(z_{1}, \ldots, z_{4^{g}}\right)$, then $\gamma(z)=y$, where $y_{i}=z_{i}$ for $i=1, \ldots, d$ and the other $y_{i}$ are determined by the linear relations on $\mathcal{Q}$. By construction, 
we have that $\beta(A)$ is contained in the domain of $\gamma$ and in fact

$$
\gamma(\beta(A)) \cong \iota(\kappa(A))
$$

But it follows from the corollary on $[\mathbf{1 7}$, p. 349] that the image of $A$ under $\beta$ is defined by an intersection of quadrics, which then must hold for $\gamma(\beta(A))$ as well, since $\gamma$ is defined by homogeneous polynomials of degree 1 . As the pullback under $\iota$ of $\gamma(\beta(A))$ is isomorphic to $K$, the result follows.

By Proposition 3.1 it suffices to find a basis for the space of quartic relations on $K$ to describe $K$. We first compute a lower bound on the dimension of this space. For $n \geqslant 1$ let $m(n)$ denote the number of monic monomials of degree $n$ in $\kappa_{1}, \ldots, \kappa_{2^{g}}$ and let $d(n)$ denote the dimension of the space spanned by them. Then we have $m(n)=\left({ }^{2^{g}+n-1}{ }_{n}\right)$. Moreover, let $e(n)$ denote the dimension of the space of even functions in $\mathcal{L}(2 n \Theta)$. By [1, Corollary 4.7.7] this is equal to $(2 n)^{g} / 2+2^{g-1}$. Since a monomial of degree $n$ in the $\kappa_{i}$ induces an even function in $\mathcal{L}(2 n \Theta)$, we always have $d(n) \leqslant e(n)$.

In genus 2 , the dimension count is given in Table 1 . We know that $d(4)$ can be at most $e(4)=34$, and indeed the space of quartic relations in $\kappa_{1}, \ldots, \kappa_{4}$ is one-dimensional, spanned by the Kummer surface equation.

In genus 3, Stubbs has found the following quadratic relation between the $\kappa_{i}$ and shown that for a generic curve it is the unique quadratic relation up to scalars:

$$
R_{1}: \kappa_{1} \kappa_{8}-\kappa_{2} \kappa_{7}-\kappa_{3} \kappa_{6}-\kappa_{4} \kappa_{5}-2 f_{5} \kappa_{2} \kappa_{4}+f_{5} \kappa_{3}^{2}+2 f_{6} \kappa_{3} \kappa_{4}+3 f_{7} \kappa_{4}^{2}=0 .
$$

The dimensions for genus 3 are presented in Table 2. The existence and uniqueness of $R_{1}$ implies that $d(2)=35$, but since $e(2)=36$, this means that there is an even function in $\mathcal{L}(4 \Theta)$ not coming from a quadratic monomial in the $\kappa_{i}$, which does not happen in genus 2 . Accordingly, we can at this point only bound $d(3) \leqslant 112$ and $d(4) \leqslant 260$ from above.

It follows that in genus 3 there must be at least $70=330-260$ quartic relations on the Kummer variety. But 36 of these are multiples of the quadratic relation $R_{1}$. Moreover, there are only eight cubic relations and they are all multiples of $R_{1}$. Hence, there must be at least 34 independent irreducible quartic relations. In [22, Chapter 5] Stubbs lists 26 quartic relations and conjectures that together with $R_{1}$ these relations are independent and form a basis of the space of all relations on the Kummer variety. His relations are at most quadratic in $\kappa_{5}, \ldots, \kappa_{8}$. Using current computing facilities we can verify the former conjecture quite easily, but because of our dimension counting argument, we know that the latter conjecture cannot hold.

TABle 1. Dimensions in genus 2.

\begin{tabular}{rrrr}
\hline$n$ & $m(n)$ & $e(n)$ & $d(n)$ \\
\hline 1 & 4 & 4 & 4 \\
2 & 10 & 10 & 10 \\
3 & 20 & 20 & 20 \\
4 & 35 & 34 & 34 \\
\hline
\end{tabular}

TABle 2. Dimensions in genus 3.

\begin{tabular}{rrrr}
\hline$n$ & $m(n)$ & $e(n)$ & $d(n)$ \\
\hline 1 & 8 & 8 & 8 \\
2 & 36 & 36 & 35 \\
3 & 120 & 112 & 112 \\
4 & 330 & 260 & 260 \\
\hline
\end{tabular}


To compute a complete set of defining equations for the Kummer variety we employ the technique already used by Stubbs. Owing to the enormous size of the algebra involved in these computations, simply searching for relations among all monomials is not feasible. Instead we split the monomials into parts of equal $x$-weight and $y$-weight. These are homogeneous weights discussed in $[\mathbf{2 2}, \S 3.5]$ that were already used by Flynn in [5] in the genus 2 situation. See Table 3 .

On monomials of equal $x$ - and $y$-weight we can use linear algebra to find relations; we continue this process with increasing weights until we have found enough quartic relations to generate a space of dimension 70 . The difficulty of this process depends essentially on the $y$-weight. We used Magma (as described in [12]; see also the Magma home page at http://magma.maths.usyd.edu.au/magma/) to find 34 relations $R_{2}, \ldots, R_{35}$ on $K$, of $y$-weight at most 8 , so that the following result holds.

Lemma 3.2. Let $C$ be defined over a field $k$ of characteristic other than $2,3,5$. Then the space

$$
\left\{R_{2}, \ldots, R_{35}\right\} \cup\left\{\kappa_{i} \kappa_{j} R_{1}: 1 \leqslant i \leqslant j \leqslant 8\right\}
$$

has dimension equal to 70 .

Proof. It suffices to show that the matrix of coefficients of the relations expressed as linear combinations of the quartic monomials in $\kappa_{i}$ has rank 70. Using Magma, we find a $70 \times 70$ minor of this matrix whose determinant is equal to the integer $2^{46} \times 3^{4} \times 5^{5}$.

These relations, as well as code for the verification alluded to in the proof, can be downloaded at [11]. One can also verify that when $\operatorname{char}(k) \in\{2,3,5\}$, the relations generate a space of dimension strictly less than 70 .

In order to show that for a given curve $C$ over a field $k$ of characteristic $\notin\{2,3,5\}$ the relations $R_{1}, \ldots, R_{35}$ actually generate all relations on the associated Kummer variety $K$, we need to show that we have $d(4)=260$ for $C$. We were unable to verify this for an arbitrary curve $C$ since the necessary manipulations seem infeasible with current computing facilities. However, by finding a single curve $C$ for which the space of cubic (respectively, quartic) homogeneous polynomials has dimension exactly 112 (respectively, 260), we can conclude that we have $d(3)=112$ and $d(4)=260$ generically. For this one can use, for instance, the curve given by $Y^{2}=Z^{8}+X^{7} Z$.

Using Proposition 3.1 we have therefore proved the following theorem.

TheOREm 3.3. Let $C: Y^{2}=F(X, Z)$ be a generic hyperelliptic curve of genus 3 defined over a field of characteristic other than 2,3,5 and having a rational Weierstrass point at $\infty$. Then the relations on the Kummer threefold are generated by the relations $R_{1}, \ldots, R_{35}$.

In practice, it is rather easy to check for a given curve whether $d(4)=260$ and we provide code for doing so at $[\mathbf{1 1}$. We have carried this out for more than 10000 curves $C$, including

TABLE 3 . The $x$-weight and $y$-weight.

\begin{tabular}{ccc}
\hline & $x$ & $y$ \\
\hline$x_{i}$ & 1 & 0 \\
$y_{i}$ & 0 & 1 \\
$f_{i}$ & $-i$ & 2 \\
$\kappa_{i}, i \leqslant 4$ & $i-1$ & 0 \\
$\kappa_{i}, i>4$ & $i-9$ & 2 \\
\hline
\end{tabular}


degenerate curves (that is, such that $\operatorname{disc}(F)=0$ ). Note that the definition of $d(n)$ makes sense for degenerate curves. In all cases we have found $d(4)=260$, so it stands to reason that this should be true in general.

Conjecture 3.4. Let $k$ be a field of characteristic other than $2,3,5$ and let $F(X, Z) \in$ $k[X, Z]$ be a binary octic form such that $\operatorname{deg}_{X}(F)=7$. Then we have $d(4)=260$.

\section{Remnants of the group law}

Keeping the notation of the previous section, we now investigate which remnants of the group law on $A$ can be exhibited on $K$. Namely, we recall results of Duquesne, show that analogues of the biquadratic form representing pseudoaddition on the Kummer surface cannot exist in our situation and prove formulas for duplication on $K$. In light of Theorem 3.3, we assume that $C$ is defined over a field $k$ of characteristic coprime to 30 . Note, however, that in the present section we do not have to assume that $d(4)=260$.

Let $T$ be a 2-torsion point on $A$. Duquesne [3, $\S$ III.2.1] has found a matrix $W_{T}$ such that projectively the identity

$$
\kappa(P+T)=W_{T} \cdot \kappa(P)
$$

holds for all $P \in A$ if we view $\kappa(P)$ and $\kappa(P+T)$ as column vectors. Duquesne's method of finding $W_{T}$ is analogous to the method employed by Flynn [6] in the genus 2 case, although there are a few additional technical difficulties. We also have that if $T \in A(k)[2]$, then $W_{T}$ is defined over $k$.

Now let $P, Q \in A$. Then in general $\kappa(P+Q)$ and $\kappa(P-Q)$ cannot be found from $\kappa(P)$ and $\kappa(Q)$, but the unordered pair $\{\kappa(P+Q), \kappa(P-Q)\}$ can be found. In other words, the map from $\operatorname{Sym}^{2}(K)$ to itself that maps $\{\kappa(P), \kappa(Q)\}$ to $\{\kappa(P+Q), \kappa(P-Q)\}$ is well defined. In fact, in the analogous situation in genus 2 there are biquadratic forms $B_{i j} \in k\left[x_{1}, \ldots, x_{4} ; y_{1}, \ldots, y_{4}\right]_{2,2}$ with the following property: if $x$ and $y$ are Kummer coordinates for $P$ and $Q$, respectively, then there are Kummer coordinates $w$ for $\kappa(P+Q)$ and $z$ for $\kappa(P-Q)$ such that

$$
w * z=B(x, y)
$$

holds. Here (4) is an abbreviation for

$$
\begin{aligned}
& B_{i j}(x, y)=w_{i} z_{j}+w_{j} z_{i} \quad \text { for } i \neq j \\
& B_{i i}(x, y)=w_{i} z_{i} .
\end{aligned}
$$

The following result says that in general such biquadratic forms cannot exist in genus 3 .

Proposition 4.1. Let $A$ be the Jacobian of a generic hyperelliptic curve $C$ of genus 3 with a $k$-rational Weierstrass point, given by an equation (1), and let $K$ be the Kummer variety associated to $A$. Then there is no set of biquadratic forms $B_{i j}(x, y)$, where $1 \leqslant i, j \leqslant 8$, satisfying the following: if $x$ and $y$ are sets of Kummer coordinates for $P, Q \in A$, respectively, then there are Kummer coordinates $w, z$ for $P+Q, P-Q$, respectively, such that (4) holds.

Proof. We can work geometrically, so we assume $k$ is algebraically closed and let $C$ be a hyperelliptic curve of genus 3 defined over $k$, given by a septic model $Y^{2}=F(X, Z)$, with Jacobian $A$. Let us fix Kummer coordinates $x(T)=\left(x(T)_{1}, \ldots, x(T)_{8}\right)$ for all $T \in A[2]$.

For each $T \in A[2]$ we get a map

$$
\pi_{T}: k\left[x_{1}, \ldots, x_{8} ; y_{1}, \ldots, y_{8}\right] \longrightarrow k\left[y_{1}, \ldots, y_{8}\right],
$$


given by evaluating the tuple $x=\left(x_{1}, \ldots, x_{8}\right)$ at $x(T)$. This induces a map

$$
\pi_{T}: \frac{k\left[x_{1}, \ldots, x_{8} ; y_{1}, \ldots, y_{8}\right]_{2,2}}{\left(R_{1}(x), R_{1}(y)\right)} \longrightarrow \frac{k\left[y_{1}, \ldots, y_{8}\right]_{2}}{\left(R_{1}(y)\right)} .
$$

Suppose a set of forms $B_{i j}(x, y), 1 \leqslant i, j \leqslant 8$, as in the statement of the proposition does exist and consider

$$
R_{1}(B):=B_{18}-B_{27}-B_{36}-B_{45}-2 f_{5} B_{24}+2 f_{5} B_{33}+2 f_{6} B_{34}+6 f_{7} B_{44} .
$$

Denote by $\overline{R_{1}(B)}$ the image of $R_{1}(B)$ in $k\left[x_{1}, \ldots, x_{8} ; y_{1}, \ldots, y_{8}\right]_{2,2} /\left(R_{1}(x), R_{1}(y)\right)$. For $T \in$ $A[2]$, arbitrary $P \in A$ and a set of Kummer coordinates $y$ for $P$, we have: if $B(x(T), y)=w * z$, then $w$ and $z$ are both Kummer coordinates for $P+T=P-T$, and thus, if $x(T)$ and $y$ are scaled suitably so that $z=w$, then we must have $B_{i j}(x(T), y)=2 z_{i} z_{j}$ for $1 \leqslant i \neq j \leqslant 8$ and $B_{i, i}(x(T), y)=z_{i}^{2}$ for $i \in\{1, \ldots, 8\}$. As an element of $K_{\mathbb{A}}$, the tuple $z$ must satisfy (3) and hence this implies

$$
\pi_{T}\left(\overline{R_{1}(B)}\right)=R_{1}(z)=0 \quad \text { for all } T \in A[2] .
$$

We claim that $\overline{R_{1}(B)}$ itself vanishes. In order to show this, we fix $T \in A[2]$ and let

$$
S(T)=\left\{s_{1}(T), \ldots, s_{36}(T)\right\}=\left\{x(T)_{i} x(T)_{j}: 1 \leqslant i \leqslant j \leqslant 8\right\} .
$$

We also fix a representative

$$
\sum_{j=1}^{8} \sum_{l=1}^{8} \lambda_{T, j, l} \cdot y_{j} \cdot y_{l}
$$

of $\pi_{T}\left(\overline{R_{1}(B)}\right)$, where

$$
\lambda_{T, j, l}=\sum_{m=1}^{36} \mu_{T, j, l, m} \cdot s_{m}(T)
$$

is linear in the $s_{m}(T)$ and we require that $\lambda_{T, 1,8}=0$, which uniquely determines our representative.

From (6) we know that we must have

$$
\lambda_{T, j, l}=0
$$

for all $j, l$ and for all $T \in A[2]$ and thus we get 64 linear equations

$$
\sum_{m}^{36} \mu_{T, j, l, m} \cdot s_{m}(T)=0 .
$$

For notational purposes, denote the elements of $A[2]$ by $\left\{T_{1}, \ldots, T_{64}\right\}$. It can be shown that the matrix $\left(s_{i}\left(T_{j}\right)\right)_{1 \leqslant i \leqslant 36,1 \leqslant j \leqslant 64}$ has generic rank equal to 35 , by showing that this holds for a particular curve $C$, for instance for $C: Y^{2}=X(X-Z)(X-2 Z)(X-3 Z)(X-4 Z)(X-$ $5 Z)(X-6 Z)$. As usual, code for this computation can be found at [11]. It follows that for a generic curve $C$, any linear relation between the $s_{i}(T)$ satisfied by all $T \in A[2]$ must be a multiple of $R_{1}\left(x(T)_{1}, \ldots, x(T)_{8}\right)$.

Suppose now that $C$ is generic, in the sense that $\left(s_{i}\left(T_{j}\right)\right)_{1 \leqslant i \leqslant 36,1 \leqslant j \leqslant 64}$ has rank 35 . Then $\left.\overline{R_{1}(B}\right)$ must vanish. The upshot of this is that if we require our $B_{i j}(x, y)$ to contain no multiples of, say, $x_{1} x_{8}$ or $y_{1} y_{8}$ as summands (which we can always arrange by applying (3)), then $R_{1}(B)=0$ follows.

Now let $P \in A$ such that $2 P$ is generic in the sense of $\S 2$ and let $x$ be a set of Kummer coordinates for $P$. Note that since $A$ is complete, such a point $P$ always exists. Because any 
set of Kummer coordinates $z$ for $P-P=0$ satisfies $z_{i}=0$ for $i=1, \ldots, 7$ and $z_{8} \neq 0$, we get $B_{i j}(x, x)=0$ for $1 \leqslant i, j \leqslant 7$ from (4). However, $R_{1}(B)=0$ implies that also $B_{18}(x, x)=0$. But for a set $w$ of Kummer coordinates for $2 P$ we have $w_{1}=B_{18}(x, x)$ up to a nonzero rational factor. This contradicts the assumption that $2 P$ is generic in the sense of $\S 2$, since for such points the first Kummer coordinate can never vanish.

REMARK 4.2. The assertion $\operatorname{rk}\left(s_{i}\left(T_{j}\right)\right)_{1 \leqslant i \leqslant 36,1 \leqslant j \leqslant 64}=35$ can be verified easily for any given curve; we have tested about 1000000 examples and found that the rank was indeed 35 in all cases. However, we were unable to show this for arbitrary $C$. For instance, it proved impossible to even write down a single $35 \times 35$ minor over the function field.

Proposition 4.1 implies that the situation is rather more complicated than in genus 2 . Recall Flynn's strategy to compute the biquadratic forms in genus 2 (see [6] or [2]): if $T \in A[2]$ and $P \in A$ is arbitrary, then we can compute

$$
\kappa_{i}(P+T) \kappa_{j}(P-T)+\kappa_{j}(P+T) \kappa_{i}(P-T)=2 \kappa_{i}(P+T) \kappa_{j}(P+T)
$$

projectively for all $i$ and $j$ by multiplying the matrix $W_{T}$ by the vector $\kappa(P) \in k^{4}$. Using some algebraic manipulations, Flynn ensures that the resulting forms $B_{i j}^{\prime}$ are biquadratic in $\kappa_{i}(P)$ and $\kappa_{j}(T)$ and satisfy some additional normalization conditions. One can then check that the space generated by all $\kappa_{i}(T) \kappa_{j}(T)$, where $i \leqslant j$, has dimension 10 . Hence, for each pair $(i, j)$, at most one biquadratic form that satisfies the same normalization conditions can specialize to $B_{i j}^{\prime}$. The crucial point is that from classical theory of theta functions we already know that biquadratic forms $B_{i j}$ satisfying (4) must exist, at least in the complex case (see Hudson's book [10]) and, thus, using the Lefshetz principle, for any algebraically closed field of characteristic zero. Therefore Flynn concludes that $B_{i j}=B_{i j}^{\prime}$ for all $i, j$.

We can try to use the same strategy in the genus 3 case. Indeed, in $[\mathbf{3}, \S$ III.2.2], Duquesne computes the correct $B_{i j}^{\prime}(x, y)$ in the special case that $x$ is a set of Kummer coordinates for $T \in A[2]$. They can be downloaded from ftp://megrez.math.u-bordeaux.fr/pub/duquesne. Because of the relation (3), we know that the space of all $\kappa_{i}(T) \kappa_{j}(T)$, where $i \leqslant j$, is not linearly independent. But we also know that it has dimension 35 , since $R_{1}$ is the only quadratic relation up to a constant factor. Now we can apply $R_{1}(x)$ and $R_{1}(y)$ to the $B_{i j}^{\prime}(x, y)$ to make sure that no terms containing, say, $x_{1} x_{8}$ or $y_{1} y_{8}$ appear and this is done by Duquesne. Thus, we can draw the same conclusion as in the genus 2 situation, namely that for each pair $(i, j)$ at most one biquadratic form that satisfies the same normalization conditions can specialize to $B_{i j}^{\prime}$. By Proposition 4.1, we know that there is no set of biquadratic forms on $K$ satisfying (4) in general. But we conjecture that we can still make use of the $B_{i j}^{\prime}$ as follows.

We define two index sets

$$
I:=\{(i, j): 1 \leqslant i \leqslant j \leqslant 8\}
$$

and

$$
E:=\{(1,8),(2,7),(3,6),(4,5),(5,5),(5,6),(5,7),(6,6)\} \subset I .
$$

We say that a pair of points $(P, Q) \in A \times A$ is good if there is a pair $\left(i_{0}, j_{0}\right) \in I \backslash E$ such that if $x$ and $y$ are Kummer coordinates for $P$ and $Q$, respectively, and $w$ and $z$ denote Kummer coordinates for $P+Q$ and $P-Q$, respectively, then we have

(i) $B_{i_{0} j_{0}}^{\prime}(x, y) \neq 0$

(ii) $w_{i_{0}} \neq 0$;

(iii) $z_{j_{0}} \neq 0$.

If $(P, Q)$ is a good pair and $x, y, w, z$ are as above, then we can normalize $w$ and $z$ so that $w_{i_{0}} z_{j_{0}}=B_{i_{0} j_{0}}^{\prime}(x, y)$. For $1 \leqslant i, j \leqslant 8$ we define $\alpha_{i, j}(x, y)$ as follows:

$$
\alpha_{i j}(x, y):=w_{i} z_{j}+w_{j} z_{i}-B_{i j}^{\prime}(x, y) .
$$


Building on a large number of numerical experiments we state a list of conjectures regarding the relations between $B_{i j}^{\prime}(x, y)$ and $w_{i} z_{j}+w_{j} z_{i}$.

Conjecture 4.3. Suppose that $(P, Q) \in A \times A$ is a good pair with respective Kummer coordinates $x$ and $y$. Then the following properties are satisfied.

(a) We have $\alpha_{i j}(x, y)=0$ for $(i, j) \in I \backslash E$.

(b) The identities

$$
-\alpha_{1,8}(x, y)=\alpha_{2,7}(x, y)=\alpha_{3,6}(x, y)=\alpha_{4,5}(x, y)
$$

and

$$
\alpha_{5,7}(x, y)=-2 \alpha_{6,6}(x, y)
$$

hold.

(c) If $\alpha_{i_{1} j_{1}}(x, y)=0$ for some $\left(i_{1}, j_{1}\right) \in E$, then all $\alpha_{i j}(x, y)$ vanish.

(d) If $\alpha_{i_{1} j_{1}}(x, y) \neq 0$ for some $\left(i_{1}, j_{1}\right) \in E$, then we have $\alpha_{i j}(x, y) \neq 0$ for all $(i, j) \in E$. If this holds and if $(i, j),\left(i^{\prime} j^{\prime}\right) \in E$, then the ratios

$$
\frac{\alpha_{i^{\prime} j^{\prime}}(x, y)}{\alpha_{i j}(x, y)}
$$

only depend on $C$ and on $(i, j),\left(i^{\prime}, j^{\prime}\right)$, but not on $x$ or $y$.

REMARK 4.4. The values $\alpha_{i j}(x, y)$ depend on the choice of the pair $\left(i_{0}, j_{0}\right) \in I \backslash E$, but note that the assertions of Conjecture 4.3 are independent of this choice.

The naive height $h$ on the Kummer surface associated to a Jacobian surface $A$ can be used to define and compute a canonical height $\hat{h}$ on $A$, which has several applications. See [7] for the construction and an algorithm for the computation of $\hat{h}$, and [20] for improvements due to Stoll. For this application, one does not have to work with the biquadratic forms $B_{i j}$, but rather with the quartic duplication polynomials $\delta$ which, however, were originally derived from the $B_{i j}$. If we assume the validity of the first two parts of Conjecture 4.3 , then we can find analogs of these polynomials in the genus 3 situation which again turn out to be quartic.

More precisely, we define

$$
\delta_{i}^{\prime}(x):=B_{i 8}^{\prime}(x, x) \in k[x] \quad \text { for } i=2, \ldots, 8,
$$

and

$$
\delta_{1}^{\prime}(x):=\frac{4 B_{18}^{\prime}(x, x)+R(x)}{3} \in k[x],
$$

where $R(x)$ is a certain quartic relation on $K$ which we use to get rid of the denominators in $\frac{4}{3} B_{18}(x, x)$. Let $\delta^{\prime}(x):=\left(\delta_{1}^{\prime}(x), \ldots, \delta_{8}^{\prime}(x)\right)$. We take the $\delta_{i}^{\prime}$ as our candidates for the duplication polynomials on $K$.

As in the genus 2 situation, we want that the set $(0, \ldots, 0,1)$ of Kummer coordinates of the origin is mapped to itself by the duplication map. This is required by the canonical height algorithms in [7] and [20]. In our situation we have

$$
\delta^{\prime}(0,0,0,0,0,0,0,1)=\left(0,0,0,0,0,0,0, f_{7}^{2}\right) .
$$

But this can be fixed easily by a simple change of models of $K$ using the map

$$
\tau\left(x_{1}, \ldots, x_{8}\right)=\left(x_{1}, \ldots, x_{7}, f_{7} x_{8}\right) .
$$

Setting

$$
\delta:=\frac{1}{f_{7}^{2}}\left(\tau \circ \delta^{\prime} \circ \tau^{-1}\right)
$$


we find

(1) $\delta_{i} \in \mathbb{Z}\left[f_{0}, \ldots, f_{7}\right]\left[x_{1}, \ldots, x_{8}\right]$ for all $i=1, \ldots, 8$;

(2) $\delta(0,0,0,0,0,0,0,1)=(0,0,0,0,0,0,0,1)$.

Based on extensive numerical evidence, we conjectured the following result in an earlier version of this article (and in [14]).

Theorem 4.5. If $P \in A$, then

$$
\delta^{\prime}(\kappa(P))=\kappa(2 P)
$$

and

$$
\delta(\tau(\kappa(P)))=\tau(\kappa(2 P))
$$

Proof. In [21], Stoll constructs polynomials which represent duplication on the image of $\xi$. It suffices to show that the map on $K$ obtained from his duplication map using the transformation in Remark 2.2 coincides with the map $\delta^{\prime}$, at least modulo the relations $R_{1}, \ldots, R_{35}$. To this end, we have used Magma, see [11].

Acknowledgements. This work grew out of Chapter 4 of my $\mathrm{PhD}$ thesis $[\mathbf{1 4}]$ at the University of Bayreuth. I would like to thank my supervisor Michael Stoll for his constant help and encouragement and for sharing a preliminary version of [21] with me. I would also like to thank Sylvain Duquesne, Victor Flynn, Damiano Testa and Tzanko Matev for helpful conversations and the referee for useful remarks. Part of this work was done while I was visiting the Université Rennes I and the University of Oxford and I thank both institutions for their hospitality.

\section{References}

1. C. Birkenhake and H. Lange, Complex Abelian varieties, 2nd edn (Springer, Berlin, 2004).

2. J. W. S. CAssels and E. V. Flynn, Prolegomena to a middlebrow arithmetic of curves of genus 2 (Cambridge University Press, Cambridge, 1996).

3. S. Duquesne, 'Calculs effectifs des points entier et rationnels sur les courbes', Thèse de doctorat, Université Bordeaux I, 2001.

4. S. Duquesne, 'Traces of the group law on the Kummer surface of a curve of genus 2 in characteristic 2', Math. Comput. Sci. 3 (2010) 173-183.

5. E. V. Flynn, 'The Jacobian and formal group of a curve of genus 2 over an arbitrary ground field', Math. Proc. Camb. Phil. Soc. 107 (1990) 425-441.

6. E. V. FLynN, 'The group law on the Jacobian of a curve of genus 2', J. reine angew. Math. 439 (1993) 45-69.

7. E. V. Flynn and N. P. Smart, 'Canonical heights on the Jacobians of curves of genus 2 and the infinite descent', Acta Arith. 79 (1997) 333-352.

8. D. Holmes, 'Computing Néron-Tate heights of points on hyperelliptic Jacobians', J. Number Theory 132 (2012) no. 2, 1295-1305.

9. D. Holmes, 'An Arakelov-Theoretic Approach to Naive Heights on Hyperelliptic Jacobians', Preprint, 2012, arXiv:math/1207.5948v2 [math.NT].

10. R. W. H. T. Hudson, Kummer's quartic surface (University Press, Cambridge, 1905).

11. Kummer threefold code, http://www.uni-oldenburg.de/fileadmin/user_upload/mathe/personen/steffen. mueller/g3kummer.zip; also included as supplementary material with the online version of this paper, http://journals.cambridge.org/sup_S1461157014000126sup001.

12. W. Bosma, J. Cannon and C. Playoust, 'The Magma algebra system I: The user language', J. Symbolic. Comput. 24 (1997) 235-265.

13. J. S. MüLLER, 'Explicit Kummer surface formulas for arbitrary characteristic', LMS J. Comput. Math. 13 (2010) 47-64.

14. J. S. MüllER, 'Computing canonical heights on Jacobians', PhD Thesis, Universität Bayreuth, 2010.

15. J. S. MÜLleR, 'Computing canonical heights using arithmetic intersection theory', Math. Comput. 83 (2014) 311-336.

16. J. S. Müller and M. Stoll, 'Canonical heights on Jacobians of genus two curves', 2014 (in preparation).

17. D. Mumford, 'On the equations defining abelian varieties. I', Invent. Math. 1 (1966) 287-354. 
18. D. Mumford, Abelian varieties, Tata Institute of Fundamental Research Studies in Mathematics 5 (Tata Institute of Fundamental Research, Bombay, 1974).

19. M. Stoll, 'On the height constant for curves of genus two', Acta Arith. 90 (1999) 183-201.

20. M. Stoll, 'On the height constant for curves of genus two, II', Acta Arith. 104 (2002) 165-182.

21. M. Stoll, An explicit theory of heights for hyperelliptic Jacobians of genus three, 2014 (in preparation) (See also http://www.mathe2.uni-bayreuth.de/stoll/talks/Luminy2012.pdf.).

22. A. G. J. Stubbs, 'Hyperelliptic curves', PhD Thesis, University of Liverpool, 2000.

\author{
J. Steffen Müller \\ Institut für Mathematik \\ Carl von Ossietzky Universität Oldenburg \\ 26111 Oldenburg \\ Germany
}

jan.steffen.mueller@uni-oldenburg.de 